\title{
Use of 3D Body Scanning Technique for Heat and Mass Transfer Modelling in Clothing
}

\author{
Agnes PSIKUTA *a, Joanna FRACKIEWICZ-KACZMAREK ${ }^{\mathrm{a}, \mathrm{b}}$, Rene M. ROSSI ${ }^{\mathrm{a}}$ \\ ${ }^{a}$ Laboratory for Protection and Physiology, Empa - Swiss Federal Laboratories for \\ Materials Science and Technology, St. Gallen, Switzerland; \\ ${ }^{b}$ Laboratory of Physics and Mechanics of Textiles (EAC 7189 CNRS/UHA), \\ University of Haute Alsace, Mulhouse, France
}

\begin{abstract}
The heat and mass transfer within the clothing system is dependent on the changing size and shape of the layers of air trapped within and on the surface of clothing. Until now, air layers in clothing were investigated only either at a discrete number of points or as the total air volume in an ensemble. However, none of the devised methods allowed the systematic, accurate and detailed evaluation of the air gap thickness nor did they address the issue of contact area in ensembles. This study extends development of the method by detailed evaluation of its reliability with regards to the scanner type, its accuracy, and scanning procedure.
\end{abstract}

Keywords: air gap, clothing contact area, 3D body scanning, heat and mass transfer in clothing

\section{Introduction}

The heat and mass transfer within the clothing system is dependent on factors associated with construction and use of a garment, such as air penetration and compression by wind, body posture and movement and clothing fit. That is mainly due to the changing size and shape of the layers of air trapped between the skin and clothing, between clothing layers alone, and in the layer adjacent to the outer surface of clothing. An air gap up to a certain thickness provides the bulk of both the thermal and evaporative resistances. As shown in figure 1 even a small change of air gap by $5 \mathrm{~mm}$ significantly increases both thermal and evaporative resistances of the clothing [1].
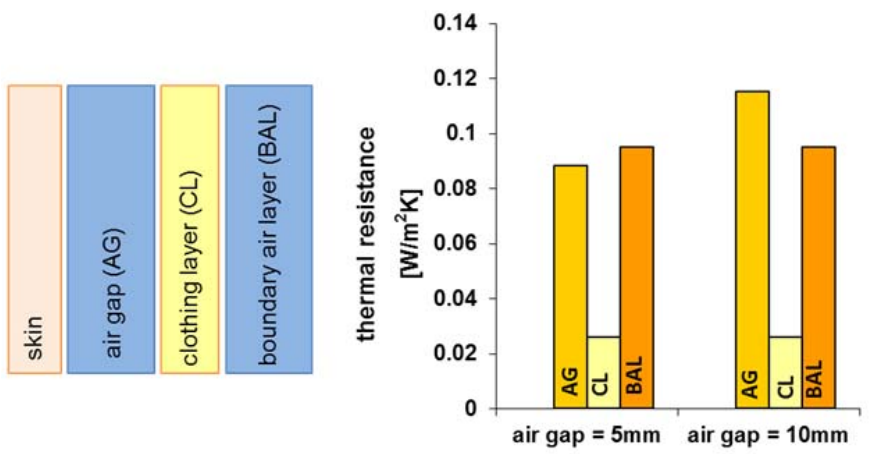

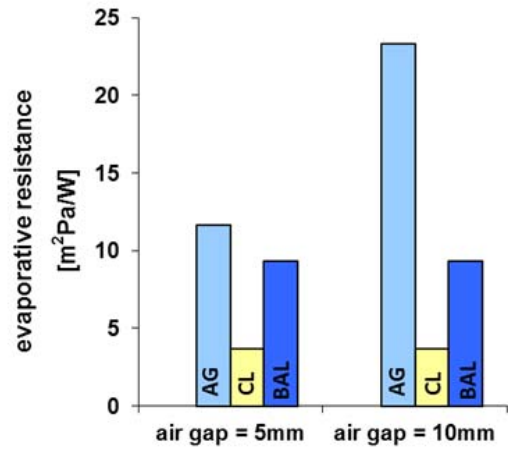

Fig. 1. Thermal and evaporative resistances of clothing layer (cotton fabric, $227 \mathrm{~g} / \mathrm{m} 2,1 \mathrm{~mm}$ thick) separated by an air gap of either $5 \mathrm{~mm}$ or $10 \mathrm{~mm}$ determined for the following conditions: Tskin $=34^{\circ} \mathrm{C}, \mathrm{RHskin}=99 \%$, Tambient $=10^{\circ} \mathrm{C}$, RHambient $=81 \%$.

For larger air gaps $(>8 \mathrm{~mm})$ [2], the local heat and water vapour exchange may be increased by the natural convection. Furthermore, when the surfaces of the fabrics and/or the surface of the skin stay in contact, the direct exchange of the liquid water will be present. Therefore, the thermal, evaporative and wicking properties of clothing depend not only on the properties of the fabric used for the garment but also on the magnitude and the temporal change of the contact area and air layer thickness. Existing mathematical clothing models assume a uniform air gap between the body and fabric layers or its full contact to facilitate the computation process [3-6]. However, this approach disregards the nonuniform heat and mass transfer due to irregular air layer $[7,8]$.

\footnotetext{
* Lerchenfeldstrasse 5, CH-9014 St. Gallen, tel: +41 587657673 email: agnes.psikuta@empa.ch
} 
Until now, air layers in clothing were investigated only either at a discrete number of points and crosssections through dressed body [9, 10] or as the total air volume in an ensemble [7] using various techniques. However, none of the devised methods allowed the systematic, accurate and detailed evaluation of the local and average air gap thickness nor did they address the issue of contact area in ensembles. Moreover, methods based on the 3D body scanning lack an extensive evaluation of the measurement uncertainty imposed by the scanner and computational algorithms. The thickness of the air gap is investigated in order of $\mathrm{mm}$ and any inaccuracies higher than that can be critical. Recently we developed at Empa a method to accurately determine a detailed surface distribution of the air gap thickness and the contact area using advanced analysis of 3D body scans [11]. This study extends development of the method by detailed evaluation of its reliability with regards to the scanner type, its accuracy, and scanning procedure.

\section{Methods}

\subsection{Manikin}

In our study a motionless male manikin (figure 2a) was scanned nude or dressed in sample clothing in standing position. To ensure a stable and reproducible position of the manikin during the test, special locks preventing movement and twisting of the body parts and a supporting construction for arms and legs were used (figure 2b).

(a)

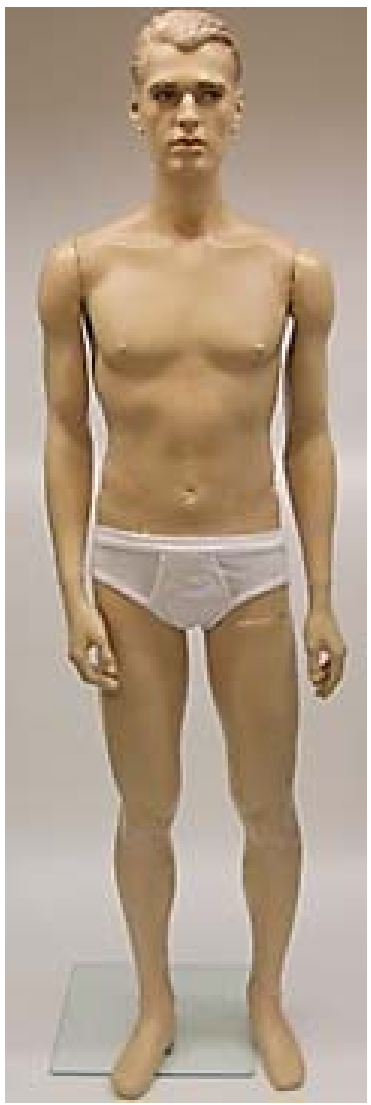

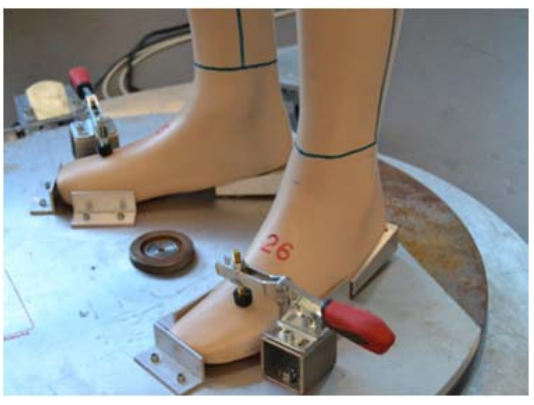

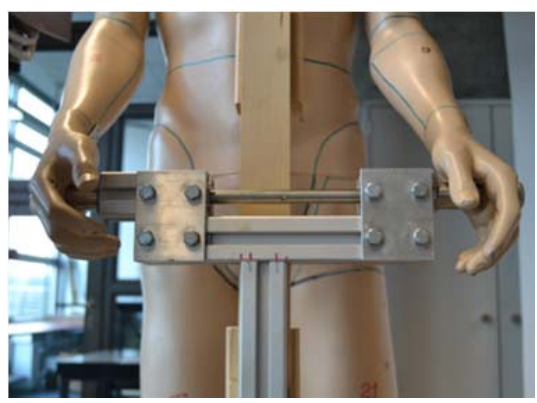

(b)

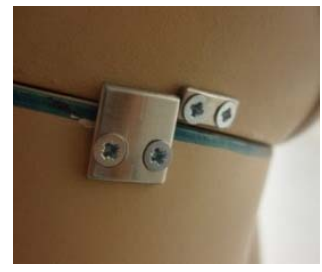

Fig. 2. Male manikin (a), and locks preventing movements and twisting of the body parts (b).

\subsection{Scanning and post-processing procedure}

Scanning and post-processing procedure consists of the following steps [11]:

- Scanning nude \& dressed manikin

- Cleaning and reorientation of the scans

- Alignment of the scans

- Geometrical determination of the distance between super-imposed surfaces recognized as air gap thickness and contact area. 
Type of the scanner, type of the scanned object \& properties of the scanned surface were chosen to be investigated for accuracy of the method as the most influential factors on the accuracy of the entire method.

\subsection{Repeatability and selection of the scanner}

The 3D whole body scanners available on the market provide scanning accuracy of up to $3 \mathrm{~mm}$ according to the manufacturers, e.g. VITUS XXL of up to $1 \mathrm{~mm}$, Artec MHT and TC ${ }^{2}$ of up to $3 \mathrm{~mm}$. On the other hand the accuracy required for investigation of thermal effects in clothing should be in order of tenth of millimetres. That is due to high insulation capacity of air (as shown in Figure 1), determination of contact area between garment and skin supporting wicking of sweat, and need for consideration of the fabric thickness. Moreover, subsequent post-processing of 3D scans may lead to further loss of scan accuracy.

In our study, the reliability of the entire method including 3D body scanning and scan post-processing was verified by determination of the alignment accuracy for two random scans of the nude manikin. In ideal case, the scans of the same manikin scanned under identical conditions should exactly match in their shapes. In other words, they are expected to show 0-mm-distance between both scan surfaces. Following the procedure for evaluation of the air gap (ref), we scanned the nude manikin ten times and determined deviations between surfaces of five randomly selected pairs. For each pair of manikin scans we calculated cumulative areas (in \% of the total area) over surface distances in $0.1 \mathrm{~mm}$ increments.

This procedure was repeated for Artec MHT (Artec Group, USA) and Vitus XXL (Human Solutions $\mathrm{GmbH}$, Germany). In opposite to the Vitus XXL scanner, the Artec MHT is designed with relatively small view area $(536 \times 371 \mathrm{~mm})$. To be able to scan the object of human size, the scanner has to be moved around the object at the defined distance band. Although scanning of large objects is possible with this small-view-area scanner, the scan accuracy is decreasing with the object size by up to $0.15 \%$ per $1000 \mathrm{~mm}$ of the exceeded dimension of the scanner view area. For the manikin with height of 1.90 $\mathrm{m}$, the expected error approximated $2.85 \mathrm{~mm}$. Also the degree of form regularity contributes significantly to scan quality due to the internal scanning and computing method of Artec MHT. Because the manikin consisted of rather regular smooth forms of its body parts a reference with geometrical features easily detectable within the scanner view area had to be added to the scanning setup. Using a reference with carefully selected size, shape and position of geometrical forms, the accuracy of the scanner was greatly improved to reach $0.6 \mathrm{~mm}$ (Figure $3 \mathrm{~b}$, the smallest distance including $100 \%$ of points).
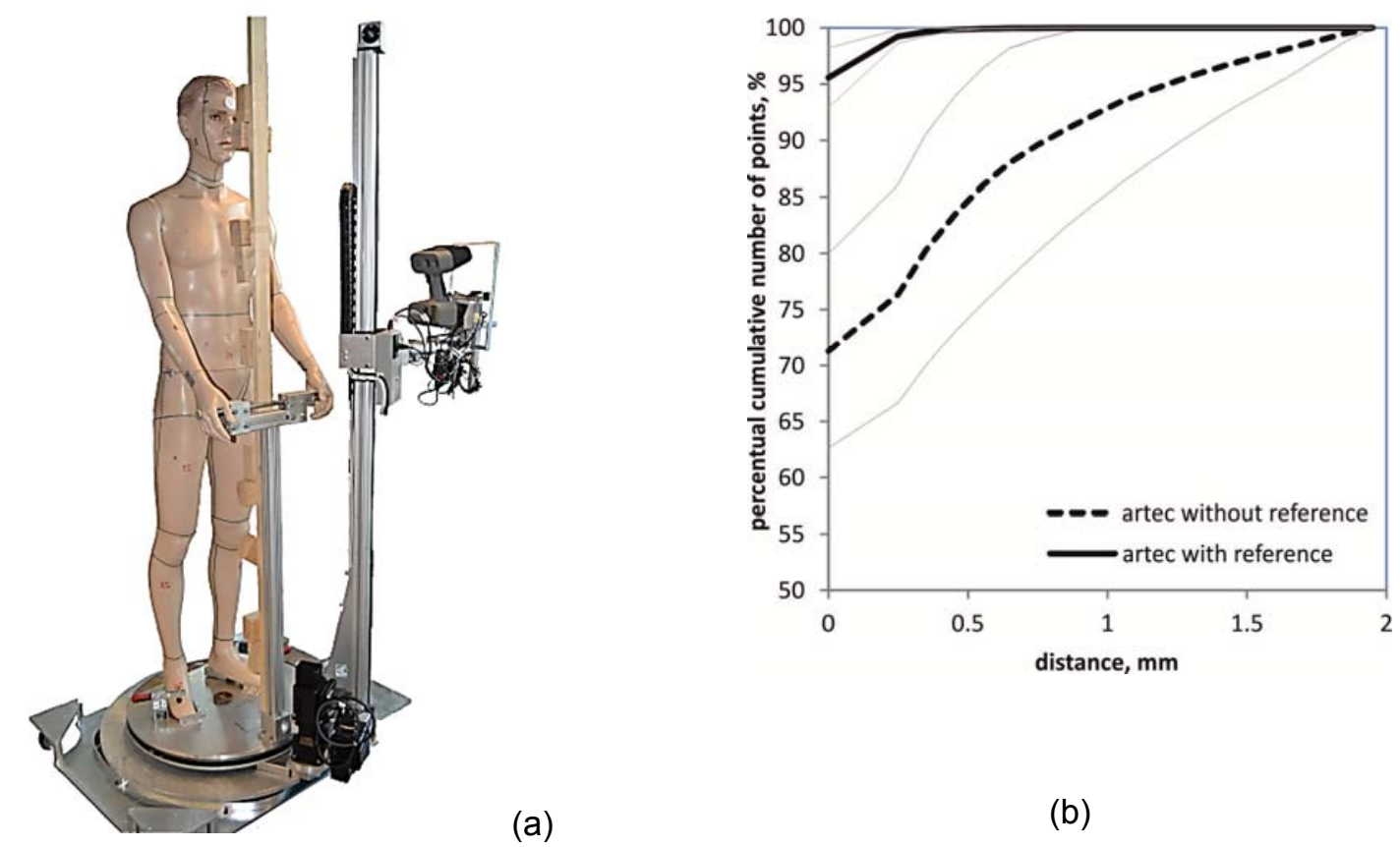

(a)

(b)

Fig. 3. Driver to move scanner to move the scanner around manikin and wooden reference geometry (a) and comparison of scan accuracy with and without the reference (grey lines indicate standard deviation). 


\subsection{Validation of the accuracy with fabric thickness}

Scanners using especially white light are known for their sensitivity to surface colour and reflectivity. Also structure of the surface may pose difficulty to the accurate scanning, for example, in case of fine fibrous structures found in fabrics. To estimate the influence of this factor on scan accuracy several fabrics with different:

- thickness - thin and thick fabrics in single and double layers,

- surface structure - smooth cotton interlock and hairy fleece,

- colour - dark khaki and light grey

were stuck onto the manikin surface using spray textile glue. The fabric pieces of about $0.1 \mathrm{~m}^{2}$ were attached on the manikin back over concave and convex areas with care to not stretch the fabric in any direction. To determine the patch thickness, the manikin was scanned with and without the fabric patch and the thickness of the fabric was derived based on surface comparison [11]. This thickness was compared to the thickness measured using Frank 16502 thicknessmeter according to standard ISO 5084 [12].

\section{Results}

\subsection{Repeatability}

Figures $4 \mathrm{a}$ and $4 \mathrm{~b}$ shows the comparison of the surface quality of scans obtained using Vitus $\mathrm{XXL}$ (with $400^{\circ} 000$ triangles/ body scan of $\sim 0.3 \mathrm{~GB}$ size) and Artec MHT (with improved accuracy over large objects and with $3^{\prime} 000^{\prime} 000$ triangles/body scan of $\sim 1 \mathrm{~GB}$ size). Figure $4 \mathrm{c}$ shows the comparison of scan accuracy of scans obtained using both scanners.

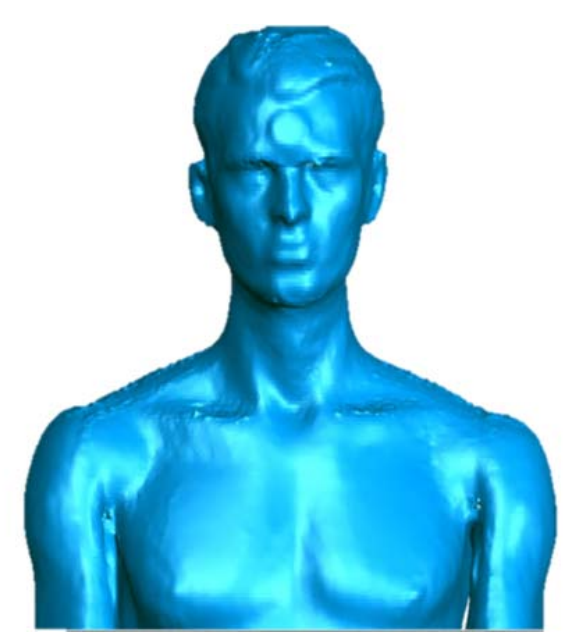

(a) Vitus XXL

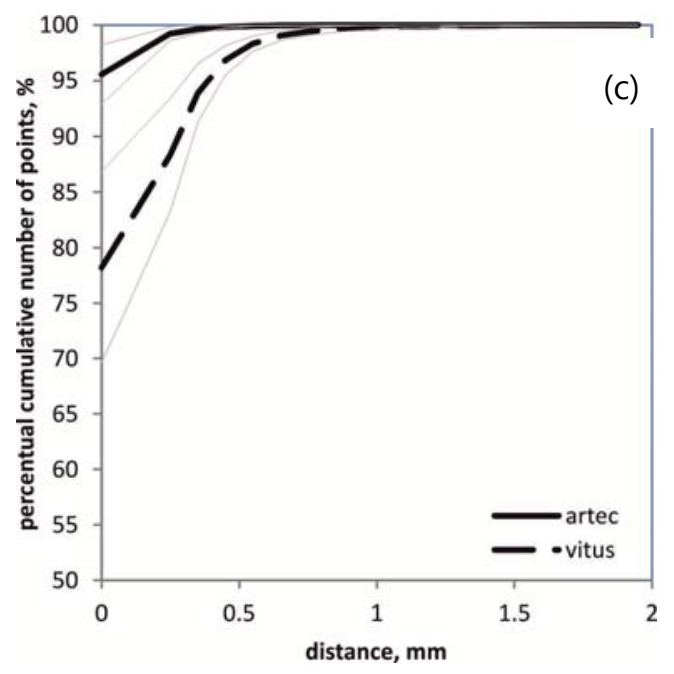

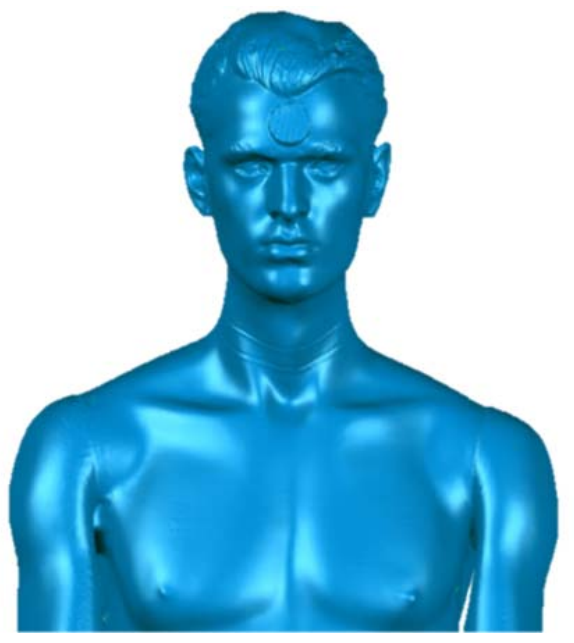

(b) Artec MHT

Fig. 4. Examples of scans obtained using Vitus XXL (a) and Artec MHT with improved accuracy over large objects (b), and the comparison of scan accuracy of scans obtained using both scanners (c) (grey lines indicate standard deviation). 


\subsection{Validation}

Figure 5 shows the comparison of the average fabric thickness measured according to the standard ISO 5084 and using Artec MHT 3D body scanner and dedicated post-processing method.

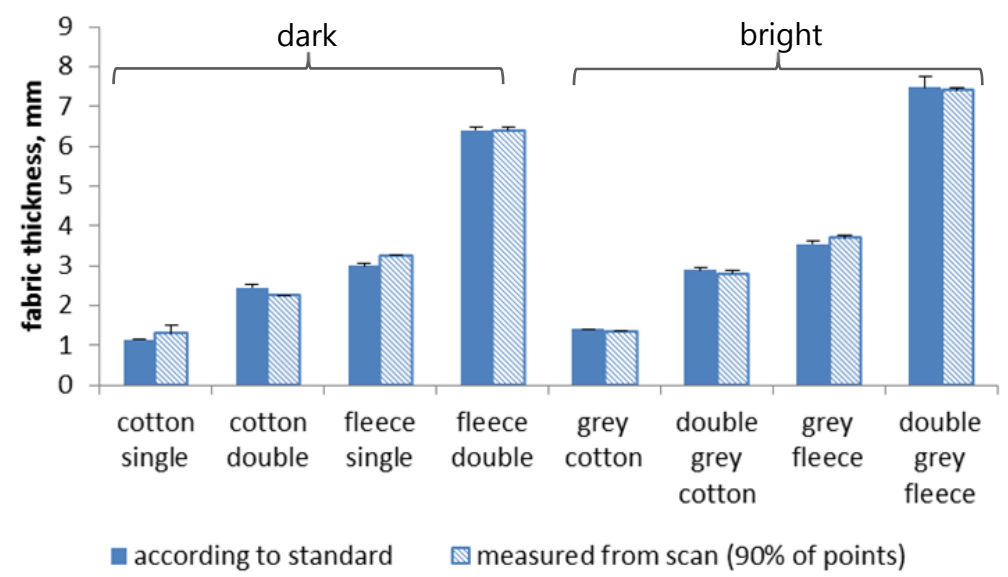

Fig. 5. Comparison of fabric thickness measured using Artec MHT 3D body scanner and dedicated postprocessing method and thickness tester according to ISO 5084.

\section{Discussion and conclusions}

Two scanners Vitus XXL and Artec MHT were compared for their performance required for the thermal modelling of clothing. Vitus XXL showed a good resultant performance with repeatable accuracy of $1 \mathrm{~mm}$. This accuracy was also reported by the manufacturer for the simple cylindrical shape which was not evident that it remains as low for a complex form of human body subjected to further postprocessing and comparison. Artec MHT showed even better performance with accuracy of $0.6 \mathrm{~mm}$, however, first after refining the scanning procedure and adding additional reference for better data processing. Thus, this scanner and methodology was chosen for further investigation.

Secondly, Artec MHT was successfully used to determine the thickness of various fabric patches attached to the convex and concaved parts of manikin back. It determined correctly the thickness of the fabric as thin as $0.9 \mathrm{~mm}$. It was also shown that the scanner is not sensitive to the surface colour (bright and dark) and structure (smooth and hairy) within the tested range.

For the first time a method to determine air layers in the garments was subjected to an extensive evaluation for accuracy. It was proven that the newly developed method, including scanning, postprocessing of scans and determining distance between skin and garment surfaces, is highly accurate $(0.6 \mathrm{~mm})$. This fact proves that this method allows accounting for the thickness of fine fabrics but not thinner than $0.6 \mathrm{~mm}$.

The developed method together with the refined scanning procedure will constitute a basis for the development of the realistic model of air gap thickness in garments. It will greatly contribute to a more realistic evaluation of heat and mass exchange rates through clothing systems and provide more accurate input for ergonomic and comfort design of clothing.

\section{Acknowledgements}

The authors wish to thank armasuisse for helping to fund this work and providing the 3D scanner. Our thanks go to the members of the workshop at Empa for their prompt support 


\section{References}

1. Wissler, E.H. and G. Havenith, A simple theoretical model of heat and moisture transport in multi-layer garments in cool ambient air. European Journal of Applied Physiology, 2009. 105(5): p. 797-808.

2. Spencer-Smith, J.L., The Physical Basis of Clothing Comfort, Part 2: Heat Transfer through Dry Clothing Assemblies. Clothing Research Journal 1977. 5(1): p. 3-17.

3. Berger, $\mathrm{X}$. and H. Sari, A new dynamic clothing model. Part 1: Heat and mass transfers. International Journal of Thermal Sciences, 2000. 39(6): p. 673-683.

4. Fan, J.T., Z.X. Luo, and Y. Li, Heat and moisture transfer with sorption and condensation in porous clothing assemblies and numerical simulation. International Journal of Heat and Mass Transfer, 2000. 43(16): p. 2989-3000.

5. $\mathrm{Li}, \mathrm{Y}$. and B.V. Holcombe, Mathematical simulation of heat and moisture transfer in a humanclothing-environment system. Textile Research Journal, 1998. 68(6): p. 389-397.

6. Lotens, W.A. and G. Havenith, Calculation of Clothing Insulation and Vapor Resistance. Ergonomics, 1991. 34(2): p. 233-254.

7. Bouskill, L., et al., The effect of external air speed on the clothing ventilation index, in Contemporary Ergonomics 1998, M.A. Hanson, Editor. 1998, Taylor \& Francis Ltd: London. p. 540-544.

8. Umeno, T., S. Hokoi, and S. Takada, Prediction of skin and clothing temperatures under thermal transient considering moisture accumulation in clothing. ASHRAE Transactions, 2001. 107(Part1, 4418): p. 71-81.

9. Wang, Z., et al., Ease distribution in relation to the X-line style jacket. Part 1: Development of a mathematical model. Journal of the Textile Institute, 2006. 97(3): p. 247-256.

10. $\mathrm{Xu}$, J.H. and W.B. Zhang, The Vacant Distance Ease Relation between Body and Garment, in ICIC2009: Second International Conference on Information and Computing Science, Vol 4, Proceedings - Modelling and Simulation in Engineering, H.G. Bao, Y. Jiang, and C. Grecos, Editors. 2009. p. 38-41.

11. Psikuta, A., et al., Quantitative evaluation of air gap thickness and contact area between body and garment. Textile Research Journal, 2012. 82(14): p. 1405-1413.

12. ISO5084:1996, Textiles - determination of thickness of textiles and textile products. 